\title{
MiR-214 Mediates Cell Proliferation and Apoptosis of Nasopharyngeal Carcinoma Through Targeting Both WWOX and PTEN
}

\author{
Ji-Bo Han, ${ }^{1}$ Mao-Ling Huang, ${ }^{1}$ Fen Li, ${ }^{2}$ Rui Yang, ${ }^{1}$ Shi-Ming Chen, ${ }^{1,2}$ and Ze-Zhang Tao ${ }^{1,2}$
}

\begin{abstract}
Background: This study aimed to investigate interactions between miR-214, PTEN, and WWOX and their effect on AKT signaling during the NPC progression. Nasopharyngeal carcinoma (NPC) was highly prevalent with poor prognosis among the patients. MiR-214 reported as an important NPC biomarker was associated with regulation of biological functions.

Methods: 5-8F and 6-10B NPC cells were transfected with miR-214 inhibitor. MTT and colony formation assays were performed to assess cell proliferation. PI staining assay was performed to determine distribution of cell cycle. Annexin-V/PI staining assay was used to evaluate cell apoptosis in NPC. The effects of miR-214 inhibitor on the expression levels of PTEN, WWOX, AKT signaling pathway, cell-cycle-, and apoptosisassociated proteins were assessed by Western blotting or qRT-PCR assay. PTEN and WWOX were knocked down using the corresponding shRNA to investigate their effects on miR-214 inhibitor involved in proapoptosis and antiproliferation mechanisms in NPC.

Results: Inhibition of miR-214 suppressed cell growth and induced apoptosis of 5-8F and 6-10B cells. MiR214 regulated the expression of both PTEN and WWOX through targeting the 3'-UTR. Inhibition of miR-214 promoted WWOX and PTEN expression, inactivated AKT signaling pathway, and regulated cell-cycle- and apoptosis-associated proteins. Knockdown of PTEN or WWOX reversed effects of miR-214 inhibitor on AKT signaling, cell proliferation, and apoptosis.

Conclusion: MiR-214 was suggested to induce cell proliferation and inhibit cell apoptosis of NPC through directly targeting both PTEN and WWOX, which provided a novel therapeutic target for clinical treatment of NPC.
\end{abstract}

Keywords: AKT signaling pathway; MiR-214; nasopharyngeal carcinoma; PTEN; WWOX

\section{Introduction}

$\mathbf{N}$ asopharyngeal carcinoma (NPC) is a malignant tumor derived from nasopharynx epithelial cells. The high prevalence of NPC occurs in Southeast Asia, South China, and Latin America. ${ }^{1,2}$ The incidence of NPC is $\sim 25$-fold higher in Southern China than in other regions of China and Western society. ${ }^{3}$ Evidence suggests that NPC is an EpsteinBarr virus (EBV)-associated malignant tumor. ${ }^{2}$ However,
EBV alone is not a sufficient reason for occurrence of this tumor. Genetic risk factors such as genetic polymorphisms in cytochrome P450 and human leukocyte antigen class I alleles, environmental exposures to low intake of fresh vegetables, and smoking are also involved in pathogenesis of NPC. ${ }^{4-6}$ Early stage NPC can be controlled by combining radiotherapy and chemotherapy clinically, while the prognosis for patients suffering relapsed and metastasized NPC still remains poor. ${ }^{7}$ Therefore, investigating novel therapeutic

\footnotetext{
${ }^{1}$ Department of Otolaryngology-Head and Neck Surgery and ${ }^{2}$ Research Institute of Otolaryngology-Head and Neck Surgery, Renmin Hospital of Wuhan University, Wuhan, People's Republic of China.

(C) Ji-Bo Han et al. 2020; Published by Mary Ann Liebert, Inc. This Open Access article is distributed under the terms of the Creative Commons Attribution Noncommercial License (http://creativecommons.org/licenses/by-nc/4.0/) which permits any noncommercial use, distribution, and reproduction in any medium, provided the original author(s) and the source are cited.
}

Address correspondence to: Ze-Zhang Tao; Department of Otolaryngology-Head and Neck Surgery, Renmin Hospital of Wuhan University, No. 238, Jie-Fang Road, Wuhan 430060, People's Republic of China E-mail: hjbjy7758521@163.com 
targets of NPC and studying its underlying molecular mechanisms are in urgent need for the purpose of better managing the NPC progression.

MicroRNAs (miRNAs) are a class of small noncoding RNAs with $\sim 20$ nt in length, which could regulate the expression of target genes by specifically binding to the $3^{\prime}$ untranslated region (UTR) of mRNAs for translational repression and degradation. ${ }^{8}$ MicroRNAs regulate complicated biological processes such as cell proliferation, invasion, and apoptosis. An increasing body of studies have indicated the regulatory roles of miRNAs involved in pathogenesis of various types of human cancers. ${ }^{9-12}$ MiR-214 has been revealed as a diagnostic or prognostic biomarker for multiple human cancers, including NPC. ${ }^{13-17}$ Notably, upregulated expression of miR-214 was found to promote the proliferation and survival of NPC through targeting lactoferrin and Bim. ${ }^{17}$ The loss of phosphatase and tensin homolog (PTEN) often causes the increased cell proliferation and the attenuated apoptosis, which occurs frequently in human cancers. ${ }^{18} \mathrm{WW}$ domain-containing oxidoreductase $(W W O X)$ gene is also a candidate tumor suppressor gene, with its reduced expression observed in NPC. ${ }^{3}$ It was indicated that the restoration of WWOX could promote cell apoptosis and autophagy against the tumor growth. ${ }^{19-21}$ Based on bioinformatics analysis and previously reported studies in other types of cancers, miR-214 could regulate the expression levels of both PTEN and WWOX. ${ }^{15,22}$ However, the crosstalk between miR-214 and PTEN or WWOX in NPC still remains unclear, and is thus worthy of further investigation.

AKT is a serine-threonine kinase that plays a central role in the PI3K/AKT/mTOR signaling pathway, which is important for promoting cell survival, proliferation, invasion, and migration, and its dysregulation frequently associates with incidence of multiple tumors. ${ }^{23-25}$ As a member of receptor-type tyrosine phosphatase (PTP), PTEN dephosphorylates PI3K and induces inactivation of the PI3K/AKT/ mTOR signaling pathway. ${ }^{26}$ For WWOX, evidence shows that it decreases phosphorylation of $\mathrm{AKT} / \mathrm{PKB}$, which is a downstream effector of PI3K, thus blocks the activation of AKT signaling and further induces cell apoptosis. ${ }^{27} \mathrm{How}-$ ever, the underlying regulatory mechanisms about the relationship between AKT signaling and PTEN or WWOX have not been fully elucidated in NPC. Therefore, we speculate that miR-214 can function to regulate AKT signaling through targeting both PTEN and WWOX, which will further mediate aberrant proliferation and apoptosis in NPC progression.

In conclusion, this study revealed the underlying mechanisms that miR-214 inhibition suppressed proliferation and antiapoptosis in NPC through targeting both PTEN and WWOX expression to mediate the downstream AKT signaling. This finding sheds substantial light on therapeutic potential of miR-214 inhibition in treating NPC clinically.

\section{Methods and Materials}

\section{Cell culture}

The 5-8F and 6-10B NPC cell lines were purchased from American Type Culture Collection (ATCC, Manassas, VA). Both NPC cell lines were maintained in Roswell Park Memorial Institute (RPMI) 1640 supplemented with $10 \%$ fetal bovine serum (Invitrogen, $\mathrm{CA}$ ) and $100 \mathrm{U} / \mathrm{mL}$ penicillinstreptomycin (Beyotime, Shanghai, China) at $37^{\circ} \mathrm{C}$ in $5 \% \mathrm{CO}_{2}$.

\section{Cell transfection}

PTEN shRNA, WWOX shRNA, miR-214 inhibitor, and their corresponding negative controls were purchased from GenePharm (Shanghai, China). MiR-214 inhibitor (hsa-miR214-3p inhibitor) is a chemically modified small, singlestranded RNA molecule that specifically inhibits mature miR-214-3p. The robust structure of the inhibitor can cause optimal miR-214 knockdown after transfection. Cells were seeded into six-well plates for $24 \mathrm{~h}$ and reached $80 \%$ confluence before the transfection. The plasmids were subsequently transfected into NPC cell lines 5-8F and 6-10B using Lipofectamine 2000 (Invitrogen) following the manufacturer's instructions. Cells were used for the following experiments $48 \mathrm{~h}$ post-transfection.

\section{3-(4,5-Dimethylthiazol-2-yl)-2,5-diphenyltetrazolium bromide assay}

Cells were resuspended into 96-well plates containing $0.2 \mathrm{~mL}$ RPMI 1640 supplemented with $10 \%$ FBS at a density of $2 \times 10^{4}$ cells per well and cultured overnight. Then, each well of the plates was administrated with $20 \mu \mathrm{L}$ of MTT solution (Sigma-Aldrich, CA), and the prepared plates were incubated for another $4 \mathrm{~h}$ at $37^{\circ} \mathrm{C}$. The supernatant was discarded, and $150 \mu \mathrm{L}$ dimethyl sulfoxide (Sigma, Shanghai, China) was added to each well. Microplate reader of SpectraMax M5 (Molecular Devices Corp., CA) was used to determine the absorbance at a wavelength of $490 \mathrm{~nm}$.

\section{Colony formation assay}

Cells were trypsinized and collected before reseeding into six-well plates at the concentration of 200 cells per well. Cells were incubated at $37^{\circ} \mathrm{C}$ for $14 \mathrm{~d}$, followed by washing with phosphate-buffered saline (PBS) and staining with crystal violet (Beyotime). Number of colonies was then calculated as reported in the previous study. ${ }^{27}$

\section{Cell-cycle analysis}

Cells were fixed in $70 \%$ ethanol for $2 \mathrm{~h}$ at $4^{\circ} \mathrm{C}$ before treating with $100 \mu \mathrm{g} / \mathrm{mL}$ RNaseA (Boehringer Mannheim, Germany) and staining with $50 \mu \mathrm{g} / \mathrm{mL}$ propidium iodium (PI; Sigma-Aldrich) for $30 \mathrm{~min}$ at $37^{\circ} \mathrm{C}$ in darkness. All samples were analyzed using an FACSCalibur flow (BD Biosciences, CA), and distribution of cell-cycle phases was measured using Modifit Software (BD Sciences). The PI value was determined based on the percentage of cells in $\mathrm{G}_{0} / \mathrm{G}_{1}, \mathrm{~S}$, and $\mathrm{G}_{2}$ phases.

\section{Annexin-V/PI assay}

Cell apoptotic rate was measured by staining cells with Annexin V-FITC and PI using Annexin V-FITC Apoptosis Detection Kit (KEYGEN, Guangzhou, China), per manufacturer's instructions. The percentage of apoptosis was measured using a Flow Cytometer (BD Biosciences). 


\section{Luciferase reporter assay}

The wild-type and mutant-type 3'-UTR segments of WWOX or PTEN mRNA that contain the miR-214 binding site were amplified, and then transfected into pGL3-basic vector (Promega, WI). The cells were seeded into 24-well plates for $24 \mathrm{~h}, 1 \times 10^{6} 5-8 \mathrm{~F}$ and $6-10 \mathrm{~B}$ NPC cells were then cotransfected with pGL3-basic vectors and miR-214 inhibitor or miR-214 NC using Lipofectamine 2000 (Invitrogen). Cells were lysed after $48 \mathrm{~h}$ of transfection, and luciferase activity was analyzed using Dual Luciferase Assay Kit (Promega). Firefly luciferase values were normalized to Renilla and presented as ratio of firefly/renilla.

\section{RNA extraction and quantitative RT-PCR}

Total RNA was extracted from cells using Trizol reagent (Invitrogen). MiRNA-214 complementary DNA (cDNA) was synthesized using One Step PrimeScript miRNA cDNA Synthesis Kit (TaKaRa, Shiga, Japan), and the High Capacity cDNA Reverse Transcription Kit (Applied Biosystems, Foster City, CA) was used for PTEN and WWOX reverse transcription. Quantitative RT-PCR was performed using SYBR Green Real-Time PCR Master Mix (TaKaRa) on the Applied Biosystems 7500 Real-Time PCR System (Thermo Fisher Scientific, MA). The GAPDH and U6 snRNA were used as internal controls. The relative expression was assessed using $2^{-\triangle \Delta C T}$ method. ${ }^{28}$ The primer sequences used were as follows:

miR-214: Forward: 5'-ATAGAATTCTTTCTCCCTTTC CCCTTACTCTCC-3';

Reverse: 5'-CCAGGATCCTTTCATAGGCACCACTCA CTTTAC-3'.

U6 snRNA: Forward: 5'-CTCGCTTCGGCAGCACA-3'; Reverse: 5'-ACGCTTCACGAATTT GCG T-3'.

WWOX: Forward: 5'-GAGTTCCTGAGCGAGTGGAC-3'; Reverse: 5'-CCCCAGGAATTCCCTGCTT-3'.

PTEN: Forward: 5'-GAGCGTGCAGATAATGACAAG GAAT-3';

Reverse: 5'-GGATTTGACGGCTCCTCTACTGTTT-3'. GAPDH: Forward: 5'-GCACCGTCAAGGCTGAGAAC-3'; Reverse: 5'-TGGTGAAGACGCCAGTGGA-3'.

\section{Western blotting analysis}

Whole proteins were extracted by lysing cells using radio immunoprecipitation lysis buffer (RIPA; Beyotime) with protease inhibitors (Roche, Diagnostics $\mathrm{GmbH}$, Mennheim, Germany). The protein content was measured using a BCA Protein Assay Kit (Bio-Rad). The equivalent amounts of proteins were then separated by $10 \%$ SDS-PAGE before transferred onto polyvinylidene difluoride membranes. The blots were blocked with 5\% nonfat milk for $1 \mathrm{~h}$ and incubated with primary antibodies against WWOX (Abcam; ab189410, 1:2000), PTEN (Abcam; ab31392, 1:1000),

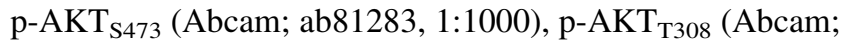
ab38449, 1:1000), AKT (Abcam; ab8805, 1:1000), cyclin D1 (Abcam; ab134175, 1:3000), cyclin-dependent kinase (CDK)2 (Abcam; ab235941, 1:2000), CDK6 (Abcam; ab151247, 1:1000), p27 (Abcam; ab32034, 1:3000), Bcl-2 (Abcam; ab196495, 1:1000), Bax (Abcam; ab53154, 1:1000), caspase-3 (Abcam; ab13847, 1:500), caspase-9 (Abcam; ab52298, 1:1000), and GAPDH (Abcam; ab181602, 1:5000) overnight at $4{ }^{\circ} \mathrm{C}$. The blots were washed and incubated with goat antirabbit IgG HRP secondary antibody (Abcam; ab6721, 1:5000) or goat antimouse (Abcam; ab97040, 1:5000) at $37^{\circ} \mathrm{C}$ for $1 \mathrm{~h}$ and visualized using ECL detection kit (Santa Cruz). The bands were quantified using Quantity One software (Bio-Rad Laboratories, Inc., Hercules, CA).

\section{Statistical analysis}

All data were expressed as the mean \pm standard deviation (SD). The difference between two groups was analyzed using $t$-test (two tailed) and one-way analysis of variance (ANOVA), followed by Tukey's post hoc test for multiple comparison. Statistical analysis was performed using Prism 6.0 (Graphpad Software). All experiments were performed at least three times in triplicate, with one representative experiment shown. A value of $p<0.05$ was considered significantly different.

\section{Results}

\section{Inhibition of miR-214 suppressed cell growth} in NPC cell lines

The 5-8F and 6-10B NPC cells were transfected with miR-214 NC or miR-214 inhibitor. The expression level of miR-214 was determined using qRT-PCR as shown in Figure 1A; the expression of miR-214 was significantly decreased in both cells transfected with miR-214 inhibitor compared with that in control or NC group. Cell proliferation was determined using MTT and colony formation assay, and the results showed inhibition of miR-214 distinctively attenuated cell proliferation and colony formation in both cell lines (Fig. 1B, C). In addition, the distribution of cells at $G_{0} / G_{1}, S$, and $G_{2}$ phases illustrated that miR-214 inhibitor increased the cell number in $G_{0} / G_{1}$ phase and decreased the cell number in $S$ and $G_{2}$ phases, which indicated that miR-214 inhibitor arrested cell cycle in $G_{1}$ phase (Fig. 1D). CDK2 as well as CDK6/cyclin D1 complex plays a key role in the $G_{1} / S$ transition, ${ }^{29}$ while cyclin-dependent kinases inhibitor p27 can negatively regulate $G_{1} / S$ phase transition. ${ }^{30}$ The results of Western blotting showed a significant decrease in the levels of cell-cycle markers CDK2, CDK6, and cyclin D1 while a remarkable elevation in the level of p27 in both cells transfected with miR-214 inhibitor (Fig. 1E). Overall, the results suggested that miR-214 suppressed cell proliferation through arresting NPC cells in $\mathrm{G}_{1}$ phase population.

\section{Inhibition of miR-214 induced cell apoptosis of NPC cell lines}

The apoptosis of both cell lines was determined by flow cytometry using Annexin V/PI double staining. Both cell lines transfected with miR-214 inhibitor showed markedly increased apoptosis ratio than those transfected with miR214 NC (Fig. 2A). As the results of Western blotting demonstrated, for both cell lines, apoptosis-related proteins cleaved caspase-3, cleaved caspase- 9 and Bax were significantly upregulated with the presence of miR-214 inhibitor while Bcl-2 was downregulated under the same condition (Fig. 2B). The results suggested that inhibition of miR-214 can induce cell apoptosis of NPC cells. 
A

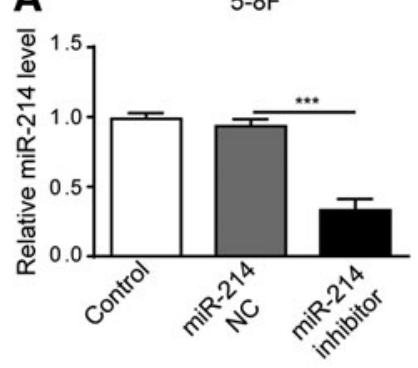

C

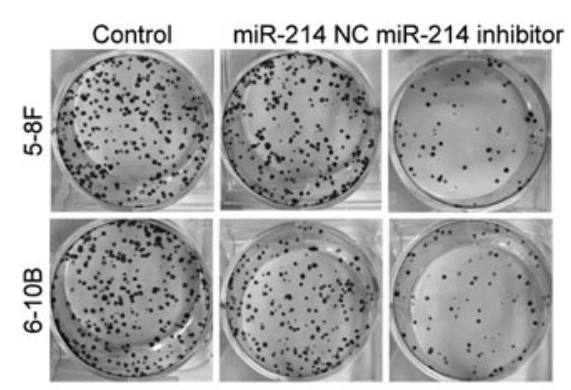

D
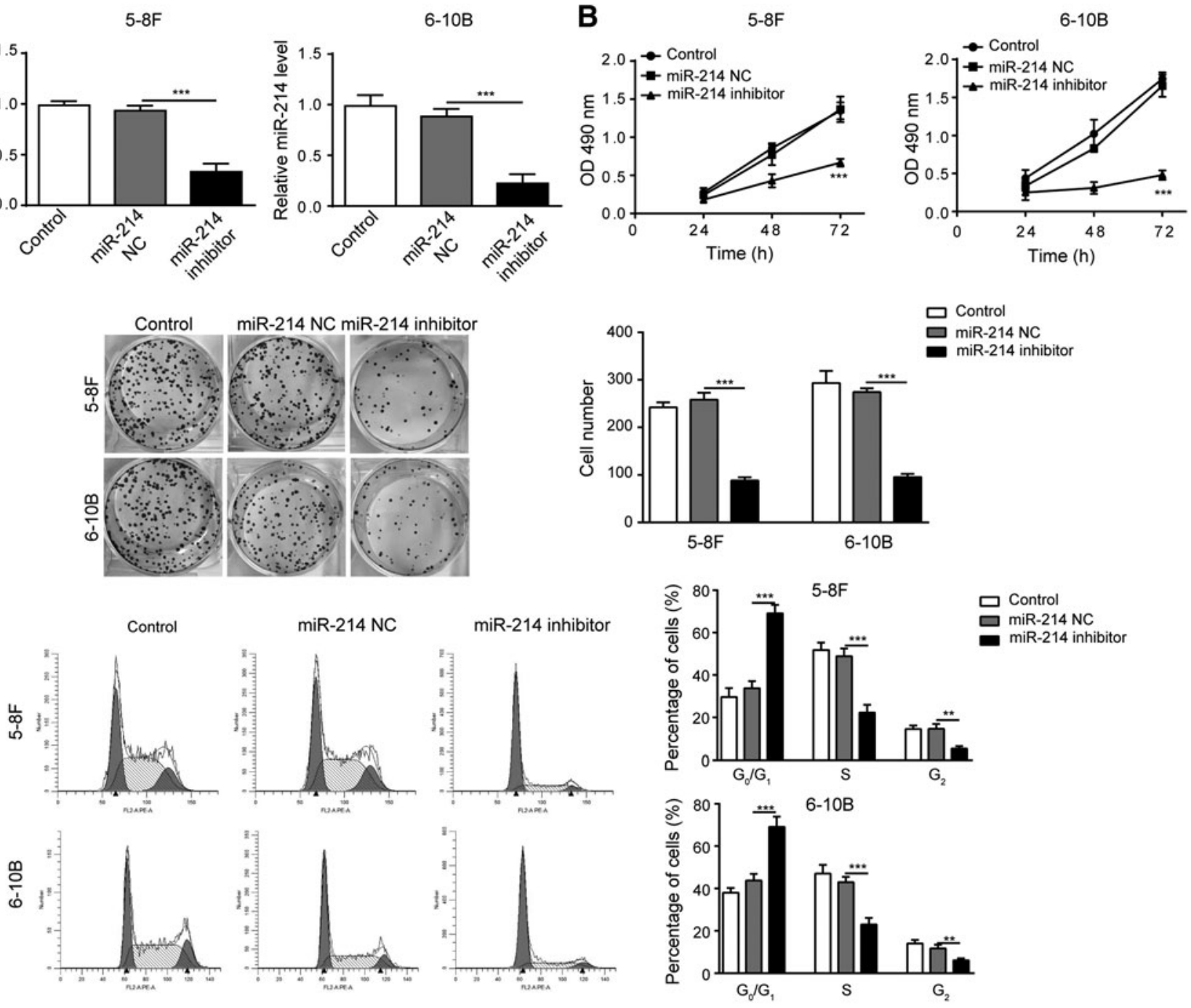
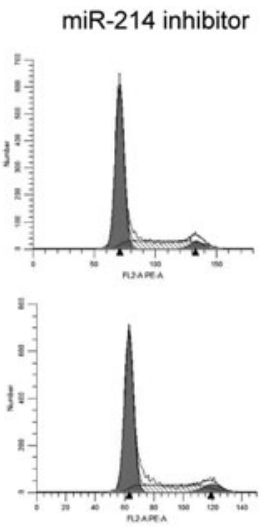

$\square$ Control

miR-214 NC miR-214 inhibitor
E

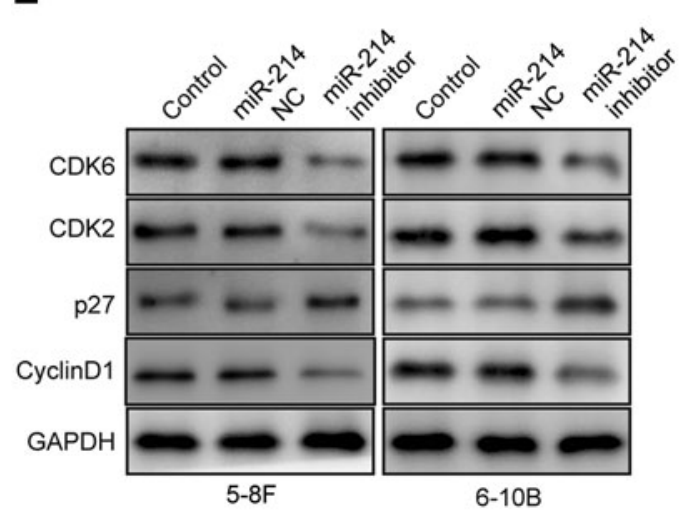

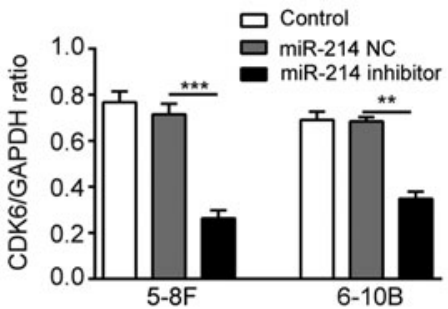

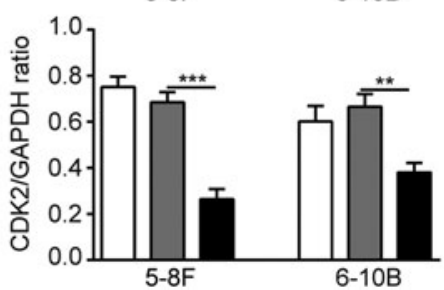

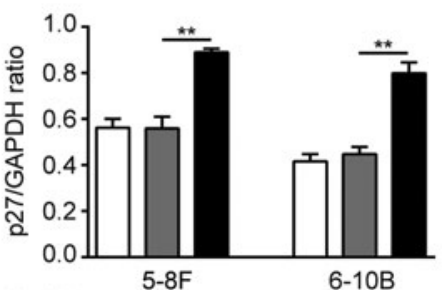

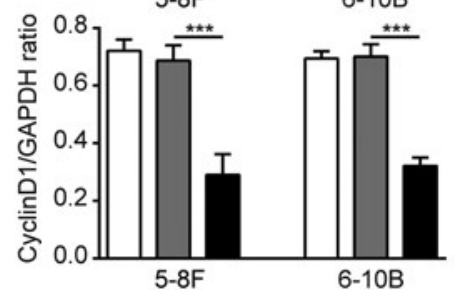

FIG. 1. Inhibition of miR-214 suppressed cell growth in NPC cell lines. (A) Relative miR-214 expression level in 5-8F and 6-10B NPC cells was measured using qRT-PCR. (B) Cell viability was measured using MTT assay after miR-214 inhibitor treatment within $72 \mathrm{~h}$. (C) Colony formation of 5-8F and 6-10B NPC cells. (D) Distribution of cell population at $\mathrm{G}_{1}, \mathrm{~S}$, and $\mathrm{G}_{2}$ phases in 5-8F and 6-10B NPC cells. (E) Cell-cycle-related proteins were measured by Western blotting. $* * * p<0.001$ and $* * p<0.01$. 
A
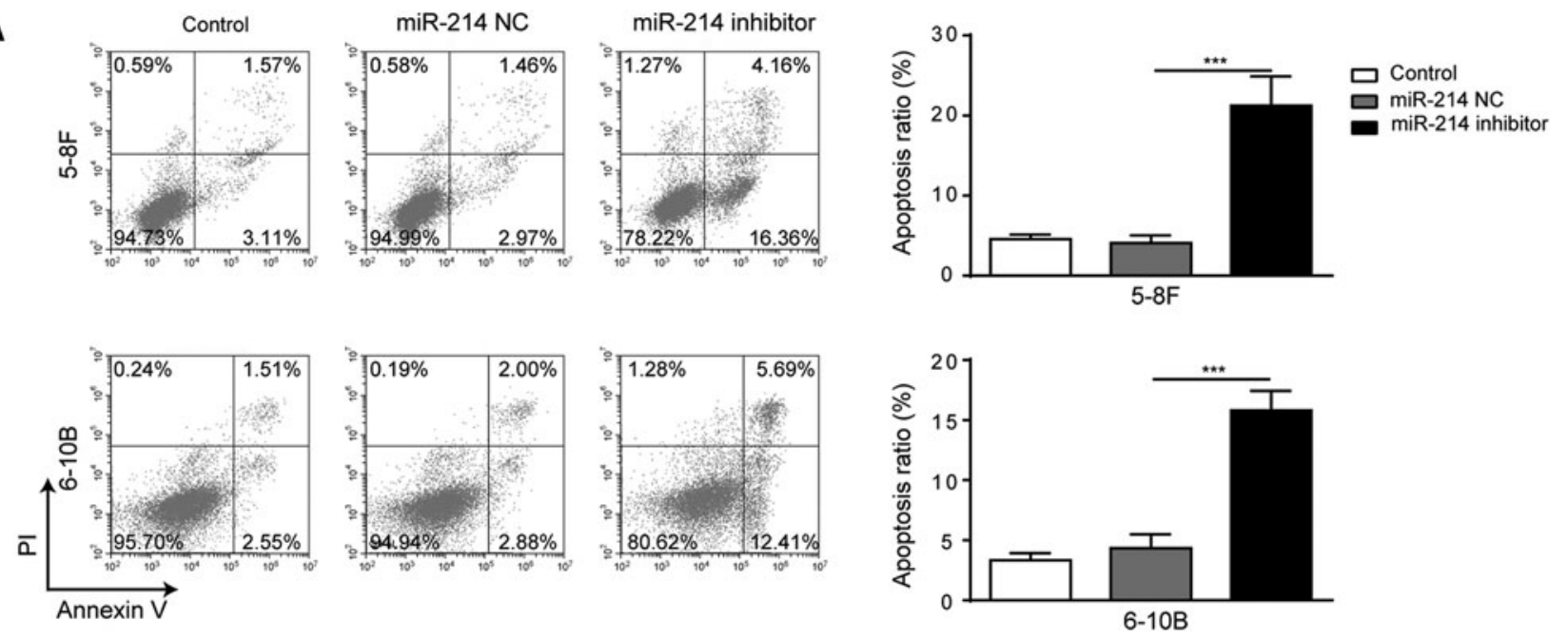
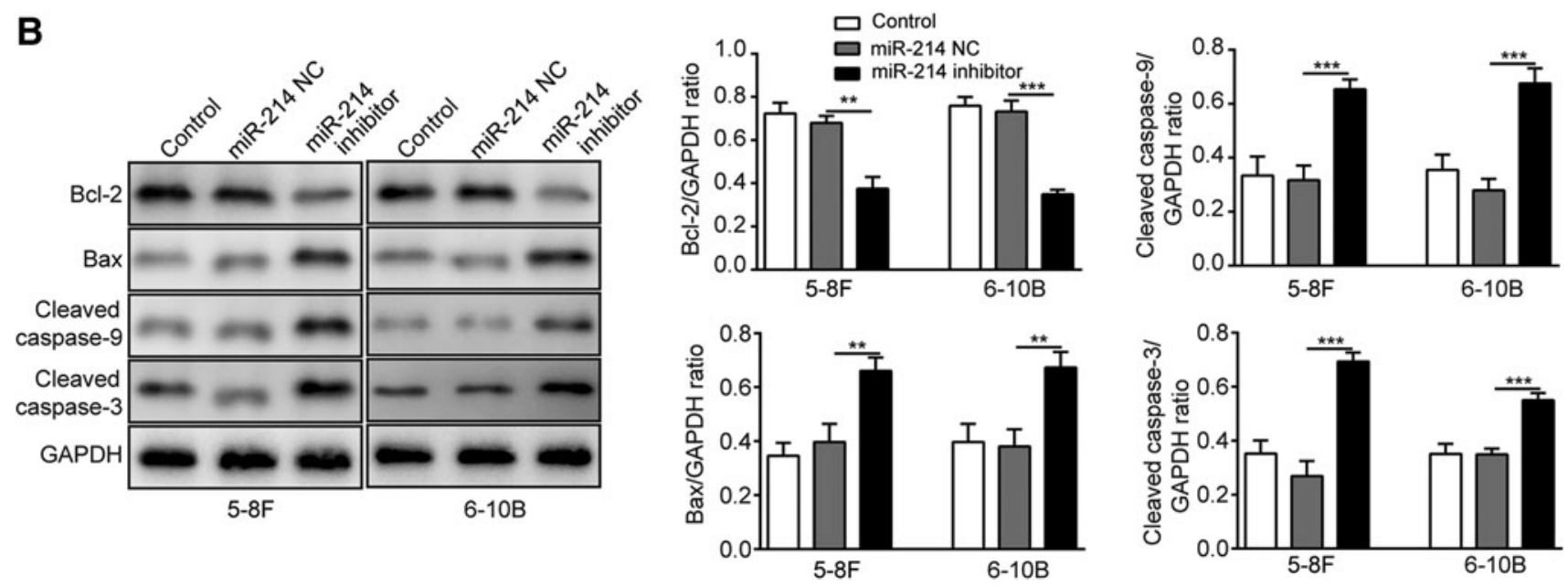

FIG. 2. Inhibition of miR-214 induced cell apoptosis of NPC cell lines. (A) Cell apoptosis rate of 5-8F and 6-10B was determined by Annexin-V/PI assay using flow cytometry. (B) Levels and quantification of cell apoptosis-related proteins after miR-214 inhibitor transfection in 5-8F and 6-10B cell lines. $* * * p<0.001$ and $* * p<0.01$.

\section{MiR-214 directly regulated the expression of PTEN and WWOX to modulate AKT signaling pathway}

The direct interactions between miR-214 and PTEN or WWOX were explored to investigate underlying mechanisms of miR-214 in NPC. The binding sites of miR-214 and PTEN or WWOX (Fig. 3A) were predicted by TargetScan website. As the results demonstrated, the relative luciferase activities in wild-type $3^{\prime}$-UTR of PTEN and WWOX were significantly higher after miR-214 inhibitor transfection. But no significant changes in mutant $3^{\prime}$-UTR of PTEN and WWOX were found (Fig. 3B). Considering that both PTEN and WWOX are involved in regulating AKT signaling, the effects of miR-214 inhibitor on protein levels of PTEN and WWOX and activity of AKT signaling pathway were also assessed. According to the results of Western blotting, the protein levels of PTEN and WWOX were significantly upregulated in $5-8 \mathrm{~F}$ and $6-10 \mathrm{~B}$ cells transfected with miR-214 inhibitor. However, the phosphorylated levels of AKT (S473 and T308) were decreased significantly with the presence of miR-214 inhibitor, which suggested the reduced AKT signaling activity (Fig. 3C). Collectively, the results showed that miR-214 inhibitor suppressed AKT signaling through direct upregulation of both PTEN and WWOX expression in NPC cells.

\section{Knockdown of PTEN or WWOX reversed the effects of miR-214 inhibitor on cell proliferation and apoptosis}

To further explore the contributions of PTEN and WWOX to biological effects of miR-214, PTEN and WWOX were knocked down in 5-8F cells to determine the impacts of PTEN and WWOX on cell proliferation and apoptosis. The silencing of PTEN and WWOX did not significantly affect the expression of miR-214 as demonstrated in Figure 4A. However, the mRNA levels of PTEN and WWOX were significantly downregulated in the corresponding knockdown groups than that in the presence of miR-214 inhibitor alone (Fig. 4A). Considering the effect of miR-214 inhibitor on upregulating PTEN and the knocking down efficiency of PTEN, it could be possible that a higher PTEN expression than the control group was observed in miR-214 inhibitor+shPTEN group. According to the results of colony 
A

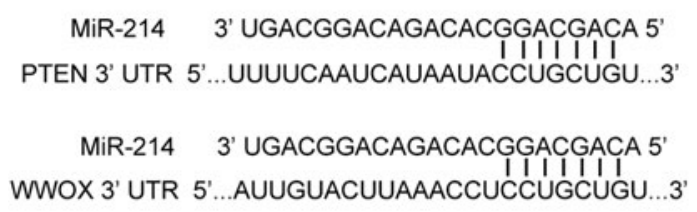

C

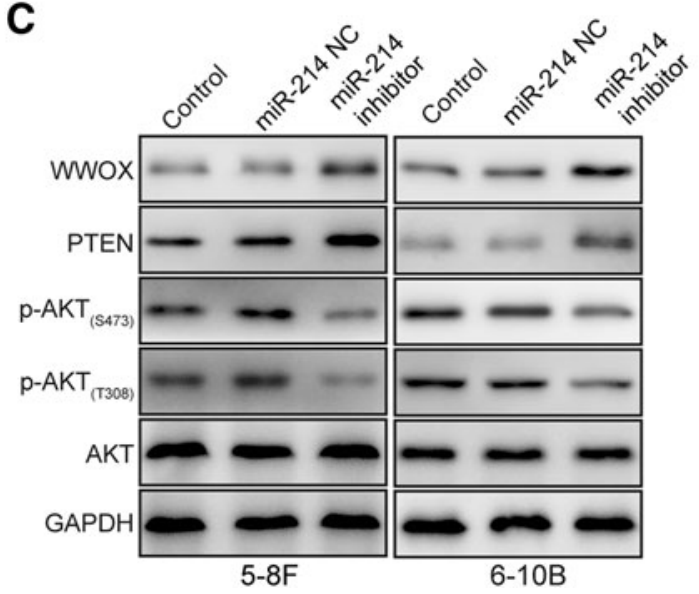

B
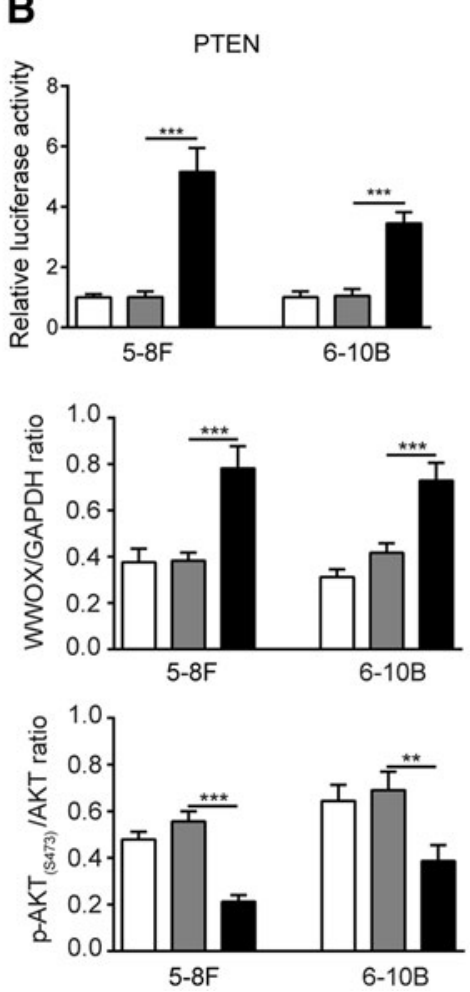
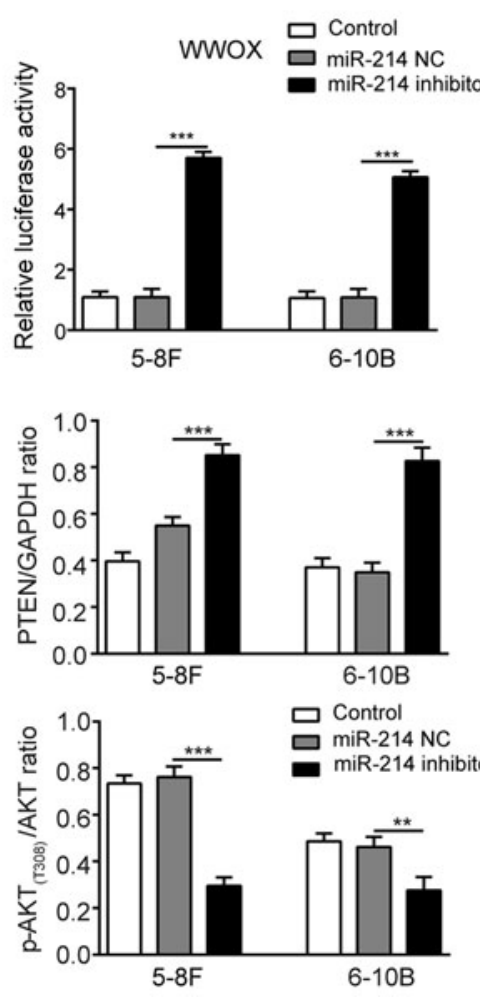

FIG. 3. MiR-214 directly regulated the expression levels of PTEN and WWOX to modulate AKT signaling pathway. (A) The binding sites between miR-214 and PTEN 3'-UTR or WWOX 3'-UTR. (B) Relative luciferase activity of PTEN 3'UTR and WWOX 3'-UTR after miR-214 inhibitor treatment. (C) Expression levels of WWOX, PTEN, AKT signalingassociated proteins after miR-214 inhibitor treatment. $* * * p<0.001$ and $* * p<0.01$.

formation assay shown in Figure 4B, the effects of miR-214 inhibitor on cell proliferation were reversed by the presence of shPTEN or shWWOX treatment. The impact of miR-214 inhibitor on distribution of cell cycle was reversed by the presence of shPTEN or shWWOX treatment based on cellcycle analysis in Figure 4C. The proapoptosis effects of miR-214 inhibitor in NPC cells were also decreased by the presence of shPTEN or shWWOX (Fig. 4D). These results demonstrated the silencing of PTEN or WWOX significantly reversed miR-214 inhibitor's proapoptosis and antiproliferation effects on 5-8F NPC cells.

\section{Knockdown of PTEN or WWOX attenuated the effects of miR-214 inhibitor on cell-cycle- and apoptosis-associated proteins and AKT signaling pathway}

To further explore the underlying molecular mechanisms responsible for regulatory effects of PTEN and WWOX on biological functions induced by miR-214, the activity of AKT signaling pathway, cell-cycle- and apoptosis-associated proteins was evaluated by Western blotting in $5-8 \mathrm{~F}$ cells. As shown in Figure 5A, the protein levels of PTEN and WWOX were upregulated in cells transfected with miR-214 inhibitor but downregulated in cells cotransfected with the corresponding shRNAs. The phosphorylated levels of AKT (S473 and T308) were reversed in cells cotransfected with miR-214 inhibitor and shPTEN or shWWOX, but no significant changes in expression of AKT were observed among different groups. The effects of miR-214 inhibition on the protein levels of cell-cycle-associated proteins (CDK6, CDK2, p27, and cyclin D1) were significantly reversed after shPTEN or shWWOX treatment (Fig. 5B). While the level of p27 was slightly upregulated based on intensity of the protein band corresponding to the miR-214+shNC group compared with the miR-214 inhibitor alone group, no significant difference was observed in terms of p27 level between two groups after the statistical analysis. The promotive effects of miR-214 inhibitor on apoptosis-associated proteins (cleaved caspase-9, cleaved caspase-3, and Bax) were abated by the silencing of PTEN or WWOX, and the attenuated expression of Bcl-2 by miR-214 inhibitor was also partly restored by the presence of shPTEN or shWWOX (Fig. 5C). The results here indicated that the silencing of PTEN or WWOX reversed the effects of miR-214 inhibitor on cell-cycle- and apoptosis-associated proteins and AKT signaling.

\section{Discussion}

NPC is commonly diagnosed in South China, Southeast Asia, and Latin America with an incidence rate ranging from 20 to 50 per 100,000 people. ${ }^{1}$ Although combination of chemotherapy and radiotherapy can effectively control the progression of NPC, the 5-year overall survival rate of the patients with advanced stage is merely $\sim 50 \%$, which has brought a heavy socioeconomic burden. ${ }^{7,31}$ Notably, miR-214 was found to be upregulated in various human tumors, including gastric cancer, ovarian cancer, and melanoma, by targeting against expressions of multiple genes 
A

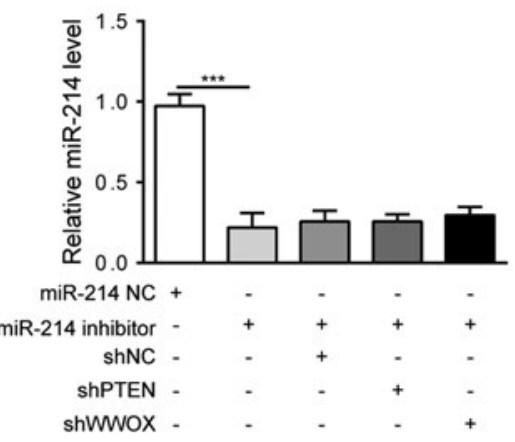

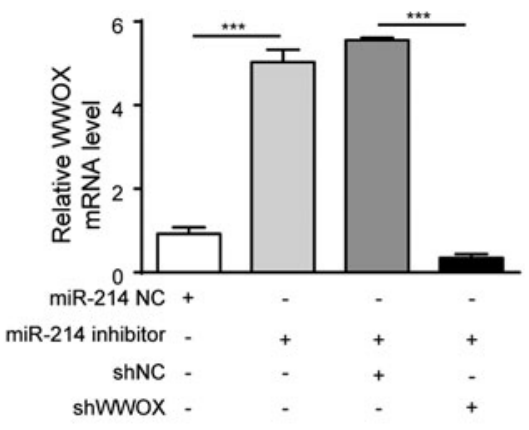

B $\begin{array}{lcccc}\text { miR-214 NC } & \text { miR-214 inhibitor } & \begin{array}{c}\text { miR-214 inhibitor } \\ + \text { shNC }\end{array} & \begin{array}{c}\text { miR-214 inhibitor+ } \\ \text { shPTEN }\end{array} & \begin{array}{c}\text { miR-214 inhibitort } \\ \text { shWWOX }\end{array}\end{array}$
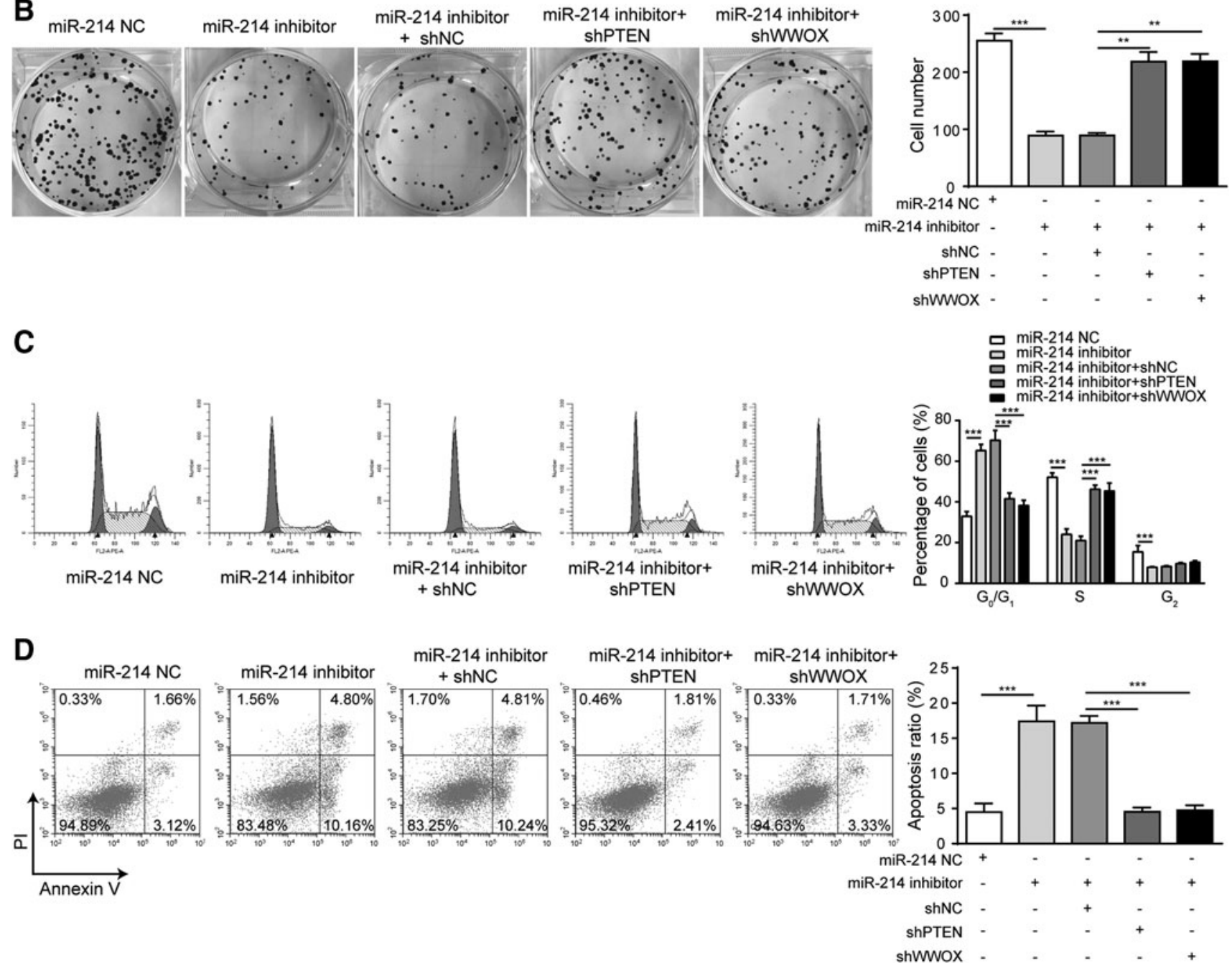

FIG. 4. Knockdown of PTEN or WWOX reversed the effects of miR-214 inhibitor on cell proliferation and apoptosis in 5-8F cell line. (A) Relative miR-214, PTEN, and WWOX levels after indicated treatment. (B) Colony formation assay was performed to test cell proliferation after indicated treatment. (C) Distribution of cell population at $\mathrm{G}_{1}, \mathrm{~S}$, and $\mathrm{G}_{2}$ phases after indicated treatment. (D) Cell apoptosis was measured in NPC cells using Annexin-V/PI assay by flow cytometry. $* * * p<0.001$ and $* * p<0.01$.

associated with tumor metastasis. ${ }^{32-34}$ This study indicated miR-214 could function to regulate AKT signaling through directly targeting both PTEN and WWOX, which further mediated aberrant proliferation and apoptosis in NPC progression. Also, miR-214 was reported to directly target both PTEN and WWOX in NPC, which is a novel finding.

Evidence has confirmed that the expression of miR-214 was significantly higher in NPC, ${ }^{12,14}$ and demonstrated that the silencing of miR-214 can enhance apoptosis, suppress cell proliferation as well as tumor growth in vivo. ${ }^{12}$ Human miR-214 gene is located in the chromosomal region 1q24.3, intron 14 of the Dynamin-3 gene. The upregulation of miR214 in NPC might be attributed to the abnormal expression of several transcriptional factors such as Twist1 or long noncoding RNAs such as lncRNA HOXA11-AS, circular RNAs such as circRNA_33287, because these factors and 
A

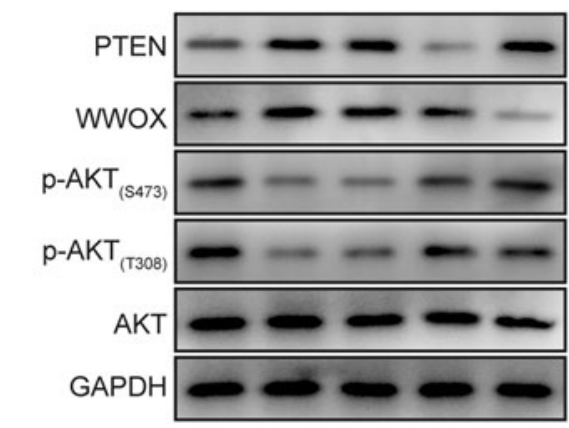

miR-214 NC

miR-214 inhibitor

shNC

ShPTEN

shWWOX

B

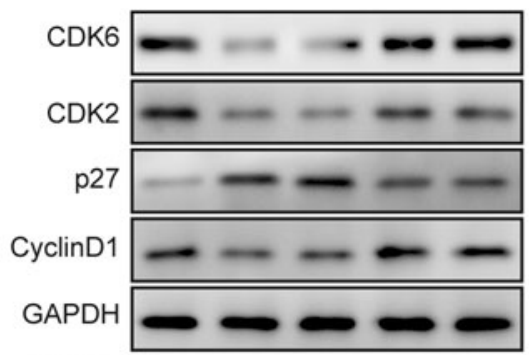

miR-214 NC +

miR-214 inhibitor

shNC -

ShPTEN -

ShWWOX -

C

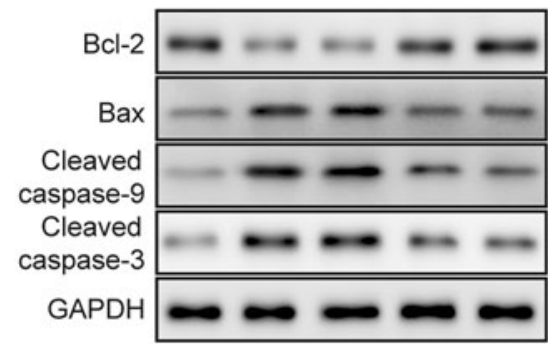

miR-214 NC

miR-214 inhibitor

shNC

ShPTEN -

shWWOX
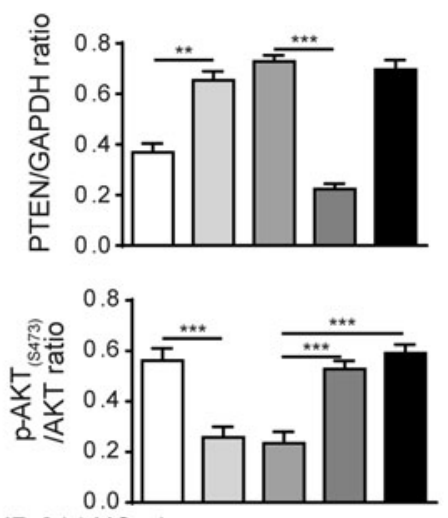

miR-214 NC

miR-214 inhibitor

shNC

ShPTEN

shWWOX
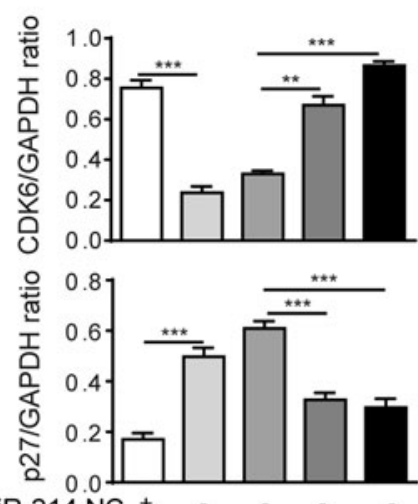

miR-214 NC

miR-214 inhibitor

shNC

ShPTEN

shWWOX
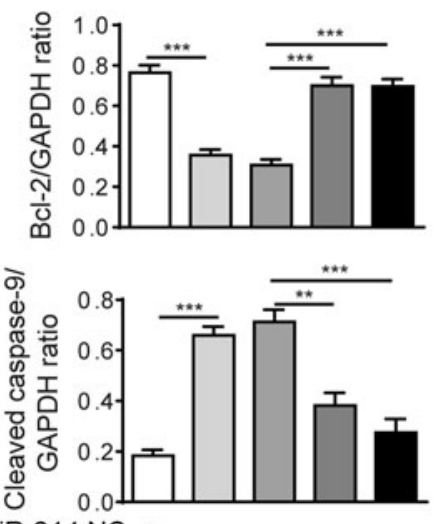

miR-214 NC

miR-214 inhibitor

shNC

ShPTEN

shWWOX
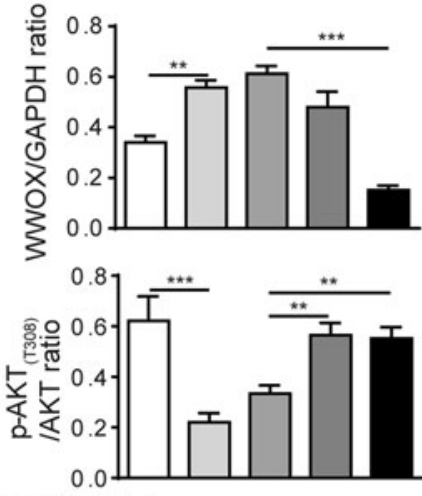

miR-214 NC +

miR-214 inhibitor -

shNC .

ShPTEN -

shWWOX -
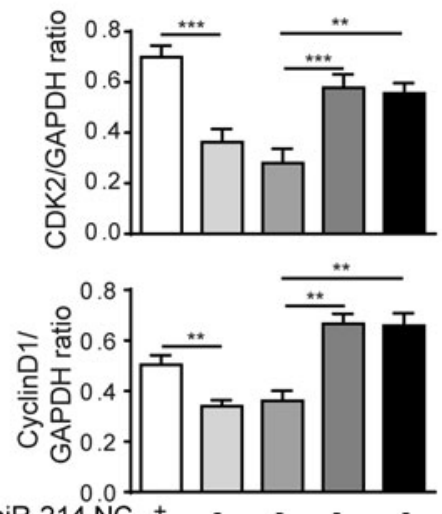

miR-214 NC

miR-214 inhibitor

shNC

ShPTEN

shWWOX
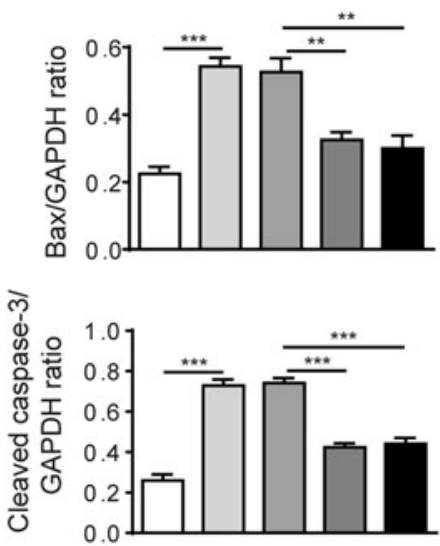

miR-214 NC

miR-214 inhibitor

ShNC

ShPTEN

shWWOX

FIG. 5. Knockdown of PTEN or WWOX attenuated the effects of miR-214 inhibitor on cell-cycle- and apoptosisassociated proteins and AKT signaling pathway in 5-8F cell line. (A) Expression levels of WWOX, PTEN, AKT signalingassociated proteins in 5-8F cells. (B) Levels of cell-cycle-associated proteins CDK2/6, p27, and cyclin D1 in 5-8F cells after indicated treatment. (C) Levels of cell-apoptosis-associated proteins in 5-8F cells after indicated treatment. $* * * p<0.001$ and $* * p<0.01$. 
noncoding RNAs could regulate the expression of miR$214,{ }^{17,32,35-37}$ which needed further investigation into NPC. Here, we investigated the role of miR-214 inhibitor in cell proliferation and apoptosis in NPC cell lines at in vitro level. In consistent with the previous studies, our study suggested that the miR-214 inhibitor attenuated cell proliferation while promoting cell apoptosis in NPC cells. Cyclin D1 binds CDK2/6 to form an active complex, which drives the initiation of the transcription of genes required for cell-cycle progression. ${ }^{38}$ The high level of cyclin D1 should be enough to trigger DNA synthesis and regulate cell-cycle transition of $\mathrm{G}_{1}$ to $\mathrm{S}$ phase. ${ }^{39}$ On the other side, p27 is a cell-cycle negative regulatory, and its overexpression can prevent CDK2 activation, which can block $G_{1}$ to $S$ phase transition. $^{40}$ The significantly downregulated cyclin D1, CDK2, and CDK6 and upregulated p27 indicated an obvious suppression of cell proliferation in NPC cells transfected with miR-214 inhibitor, which resulted from the cell-cycle arrest at the $G_{1}$ phase. Moreover, apoptosis-related marker caspase- 3 is an effector for caspase-9, and its activation is the central event for the apoptosis progression. ${ }^{41}$ The elevated levels of Bax, cleaved caspase- 9 and cleaved caspase3 and decreased Bcl-2 expression confirmed that inhibition of miR-214 promoted apoptosis of NPC cells.

PTEN and WWOX both were reported as tumor suppressor genes, and their expression was suggested to be directly regulated by miR-214 in our study. PTEN was previously reported as tumor suppressor, and its aberrant expression was involved in multiple cancers, including NPC. ${ }^{15,42,43}$ Earlier studies have reported that miR-214 can regulate tumor progression through directly targeting PTEN expression in osteosarcoma. ${ }^{44}$ For WWOX, evidence showed that the downregulation of WWOX was frequent in NPC, ${ }^{3}$ and increased WWOX expression was indicated to be corresponding to the attenuated phosphorylation level of AKT in tumors, such as hepatocellular carcinoma. ${ }^{45}$ Notably, though the regulatory effects of miR-214 on WWOX expression were reported in osteosarcoma, ${ }^{22}$ however, it has not been unraveled whether there is the same mechanism in NPC cells. Our study revealed that miR-214 directly regulated expression of both PTEN and WWOX through binding to their $3^{\prime}$-UTR by establishing luciferase reporter system in NPC cells for the first time. Also, miR-214 inhibitor significantly promoted the protein levels of PTEN and WWOX, suggesting the inhibitory effect of miR-214 on expression of WWOX and PTEN in NPC cells. While the previous studies have indicated solo regulatory effect of miR-214 on PTEN or WWOX, ${ }^{15,22,44}$ to our knowledge, this is for the first time that miR-214 is reported to regulate both PTEN and WWOX in NPC. Our study also demonstrated that PTEN and WWOX simultaneously regulated AKT signaling, which was not mentioned in the previous research. In addition, the previous studies did not comprehensively evaluate the impacts on cell proliferation and apoptosis using MTT assay, colony formation, and flow cytometry assay together. Meanwhile, we validated the results by measuring protein levels of relevant cell-cycle- and apoptosis-associated markers by Western blotting, which illustrated the superiority of this study.

By targeting PTEN and WWOX, the inhibition of miR214 was indicated to suppress AKT signaling in NPC cells at in vitro level in this study. The phosphorylation level of AKT was decreased significantly with the presence of miR-214 inhibitor based on our findings. AKT is a serine/ threonine (Ser/Thr) protein kinase that plays key roles in the regulation of cellular signaling pathways, and its activation is associated with protection against apoptosis. ${ }^{46,47}$ In addition, AKT activation affects cell-cycle progression through regulation of cyclin D stability and inhibition of p27 protein levels, ${ }^{48,49}$ which have been studied in our study. So, here we did not investigate the role of other downstream factors and gene products that might be involved in the molecular mechanism of apoptosis and proliferation in NPC. Consistent with the previous findings in other types of cancers, ${ }^{15,17}$ this study suggested that miR214 could promote AKT signaling to modulate the cell proliferation and apoptosis through inhibiting PTEN and WWOX in NPC.

Both PTEN and WWOX were proved to be the downstream effectors of miR-214 in this study. The knockdown of PTEN or WWOX has not caused a significant alteration on miR-214 expression in NPC cells. However, it reversed antiproliferation and proapoptosis functions of miR-214 inhibitor on NPC cells, which was shown in this study. Furthermore, the underlying molecular mechanism revealed that the cotransfection of miR-214 inhibitor and shPTEN or shWWOX dramatically downregulated PTEN or WWOX, respectively. Considering that both PTEN and WWOX were demonstrated to be downregulated in NPC cells, we demonstrated that both PTEN and WWOX were the downstream molecules of miR-214. Moreover, our findings suggested that both PTEN and WWOX could regulate AKT signaling due to the loss of AKT activity caused by miR-214 inhibitor, which was reversed after silencing PTEN or WWOX in NPC cells.

\section{Conclusion}

In conclusion, this study confirmed the effects of miR-214 on regulating cell proliferation and apoptosis through downregulating both PTEN and WWOX through directly binding to their $3^{\prime}$-UTR. Moreover, we revealed the underlying mechanism that AKT signaling pathway was involved in this process. However, limitations still exist in this study. This study is restricted to in vitro level, and thus future study is required to extend the current findings to in vivo level. Our findings suggest important roles of miR-214 in regulating PTEN and WWOX-mediated AKT signaling, which provide a new insight into securing therapeutic targets in treating NPC clinically.

\section{Disclosure Statement}

The authors declare that they do not have conflict of interest.

\section{Funding Information}

This research was funded by the National Natural Science Foundation of China (81372880).

\section{References}

1. Oluwadara O, Barkhordarian A, Giacomelli L, et al. Immune surveillance of nasopharyngeal carcinoma (NpC). Bioinformation 2011;7:271. 
2. Bruce JP, Yip K, Bratman SV, et al. Nasopharyngeal Cancer: Molecular Landscape. J Clin Oncol 2015;33:3346.

3. Yang Z, Lan H, Chen X, et al. Molecular alterations of the WWOX gene in nasopharyngeal carcinoma. Neoplasma 2014;61:170.

4. Liu YT, Dai JJ, Xu CH, et al. Greater intake of fruit and vegetables is associated with lower risk of nasopharyngeal carcinoma in Chinese adults: A case-control study. Cancer Causes Control 2012;23:589.

5. Xue WQ, Qin HD, Ruan HL, et al. Quantitative association of tobacco smoking with the risk of nasopharyngeal carcinoma: A comprehensive meta-analysis of studies conducted between 1979 and 2011. Am J Epidemiol 2013;178:325.

6. Tang M, Lautenberger JA, Gao X, et al. The principal genetic determinants for nasopharyngeal carcinoma in China involve the HLA class I antigen recognition groove. PLoS Genet 2012;8:e1003103.

7. Yoshizaki T, Ito M, Murono S, et al. Current understanding and management of nasopharyngeal carcinoma. Auris Nasus Larynx 2012;39:137.

8. Valencia-Sanchez MA, Liu J, Hannon GJ, et al. Control of translation and mRNA degradation by miRNAs and siRNAs. Genes Dev 2006;20:515.

9. Ahmed FE. Role of miRNA in carcinogenesis and biomarker selection: A methodological view. Expert Rev Mol Diagn 2007;7:569.

10. Hiyoshi Y, Kamohara H, Karashima R, et al. MicroRNA21 regulates the proliferation and invasion in esophageal squamous cell carcinoma. Clin Cancer Res 2009;15:1915.

11. Moriyama T, Ohuchida K, Mizumoto K, et al. MicroRNA21 modulates biological functions of pancreatic cancer cells including their proliferation, invasion, and chemoresistance. Mol Cancer Ther 2009;8:1067.

12. Zhang ZC, Li YY, Wang HY, et al. Knockdown of miR214 promotes apoptosis and inhibits cell proliferation in nasopharyngeal carcinoma. PLoS One 2014;9:e86149.

13. Lu S, Gao Y, Huang X, et al. Cantharidin exerts antihepatocellular carcinoma by miR-214 modulating macrophage polarization. Int J Biol Sci 2014;10:415.

14. Deng M, Ye Q, Qin Z, et al. miR-214 promotes tumorigenesis by targeting lactotransferrin in nasopharyngeal carcinoma. Tumour Biol 2013;34:1793.

15. Yang TS, Yang XH, Wang XD, et al. MiR-214 regulate gastric cancer cell proliferation, migration and invasion by targeting PTEN. Cancer Cell Int 2013;13:68.

16. Wang Z, Cai H, Lin L, et al. Upregulated expression of microRNA-214 is linked to tumor progression and adverse prognosis in pediatric osteosarcoma. Pediatr Blood Cancer 2014;61:206.

17. Penna E, Orso F, Taverna D. miR-214 as a key hub that controls cancer networks: Small player, multiple functions. J Invest Dermatol 2015; 135:960.

18. Chu EC, Tarnawski AS. PTEN regulatory functions in tumor suppression and cell biology. Med Sci Monit 2004;10: $\mathrm{Ra} 235$.

19. Bonin F, Taouis K, Azorin P, et al. VOPP1 promotes breast tumorigenesis by interacting with the tumor suppressor WWOX. BMC Biol 2018;16:109.

20. Lo JY, Chou YT, Lai FJ, et al. Regulation of cell signaling and apoptosis by tumor suppressor WWOX. Exp Biol Med (Maywood) 2015;240:383.

21. Tsai CW, Lai FJ, Sheu HM, et al. WWOX suppresses autophagy for inducing apoptosis in methotrexate-treated human squamous cell carcinoma. Cell Death Dis 2013;4:e792.
22. Gao K, Yin J, Dong J. Deregulated WWOX is involved in a negative feedback loop with microRNA-214-3p in osteosarcoma. Int J Mol Med 2016;38:1850.

23. Ebrahimi S, Hosseini M, Shahidsales S, et al. Targeting the Akt/PI3K Signaling Pathway as a Potential Therapeutic Strategy for the Treatment of Pancreatic Cancer. Curr Med Chem 2017;24:1321.

24. Yang WB, Zhang WP, Shi JL, et al. MiR-4299 suppresses non-small cell lung cancer cell proliferation, migration and invasion through modulating PTEN/AKT/PI3K pathway. Eur Rev Med Pharmacol Sci 2018;22:3408.

25. Wu S, Wang Y, Yuan Z, et al. Human adiposederived mesenchymal stem cells promote breast cancer MCF7 cell epithelialmesenchymal transition by cross interacting with the TGFbeta/Smad and PI3K/AKT signaling pathways. Mol Med Rep 2019;19:177.

26. Castellino RC, Durden DL. Mechanisms of disease: The PI3K-Akt-PTEN signaling node - an intercept point for the control of angiogenesis in brain tumors. Nat Clin Pract Neurol 2007;3:682.

27. Hu BS, Tan JW, Zhu GH, et al. WWOX induces apoptosis and inhibits proliferation of human hepatoma cell line SMMC-7721. World J Gastroenterol 2012;18:3020.

28. Livak KJ, Schmittgen TD. Analysis of relative gene expression data using real-time quantitative PCR and the 2(-Delta Delta C(T)) Method. Methods 2001;25:402.

29. Peng C, Zeng W, Su J, et al. Cyclin-dependent kinase 2 (CDK2) is a key mediator for EGF-induced cell transformation mediated through the ELK4/c-Fos signaling pathway. Oncogene 2015;35:1170.

30. Møller MB. p27 in Cell Cycle Control and Cancer. Leuk Lymphoma 2000;39:19.

31. Bhattacharyya N. The impact of race on survival in nasopharyngeal carcinoma: A matched analysis. Am J Otolaryngol 2004;25:94.

32. Yin G, Chen R, Alvero AB, et al. TWISTing stemness, inflammation and proliferation of epithelial ovarian cancer cells through MIR199A2/214. Oncogene 2010;29:3545.

33. Ueda T, Volinia S, Okumura H, et al. Relation between microRNA expression and progression and prognosis of gastric cancer: A microRNA expression analysis. Lancet Oncol 2010;11:136.

34. Penna E, Orso F, Cimino D, et al. microRNA-214 contributes to melanoma tumour progression through suppression of TFAP2C. EMBO J 2011;30:1990.

35. Lee YB, Bantounas I, Lee DY, et al. Twist-1 regulates the miR-199a/214 cluster during development. Nucleic acids Res 2009;37:123.

36. Wang X, Li H, Shi J. LncRNA HOXA11-AS Promotes Proliferation and Cisplatin Resistance of Oral Squamous Cell Carcinoma by Suppression of miR-214-3p Expression. Biomed Res Int 2019;2019:8645153.

37. Peng W, Zhu S, Chen J, et al. Hsa_circRNA_33287 promotes the osteogenic differentiation of maxillary sinus membrane stem cells via miR-214-3p/Runx3. Biomed Pharmacother 2019;109:1709.

38. DeGregori J. The Rb network. J Cell Sci Jul 15 2004;117: 3411.

39. Qie S, Diehl JA. Cyclin D1, cancer progression, and opportunities in cancer treatment. J Mol Med (Berl) 2016;94:1313.

40. Tian YF, Wang HC, Luo CW, et al. Preprogramming therapeutic response of $\mathrm{PI} 3 \mathrm{~K} / \mathrm{mTOR}$ dual inhibitor via the regulation of EHMT2 and p27 in pancreatic cancer. Am J Cancer Res 2018;8:1812. 
41. Sakai T, Liu L, Teng X, et al. Nucling recruits Apaf-1/ pro-caspase- 9 complex for the induction of stress-induced apoptosis. J Biol Chem 2004;279:41131.

42. Gao Q, Tang L, Wu L, et al. LASP1 promotes nasopharyngeal carcinoma progression through negatively regulation of the tumor suppressor PTEN. Cell Death Dis 2018;9: 393.

43. Xin R, Bai F, Feng Y, et al. MicroRNA-214 promotes peritoneal metastasis through regulating PTEN negatively in gastric cancer. Clin Res Hepatol Gastroenterol 2016; 40:748.

44. Liu CJ, Yu KL, Liu GL, et al. MiR-214 promotes osteosarcoma tumor growth and metastasis by decreasing the expression of PTEN. Mol Med Rep 2015;12:6261.
45. Xie B, Zen Q, Wang X, et al. ACK1 promotes hepatocellular carcinoma progression via downregulating WWOX and activating AKT signaling. Int J Oncol 2015;46:2057.

46. Franke TF, Hornik CP, Segev L, et al. PI3K/Akt and apoptosis: Size matters. Oncogene 2003;22:8983.

47. Zhang X, Tang N, Hadden TJ, et al. Akt, FoxO and regulation of apoptosis. Biochim Biophys Acta 2011;1813: 1978.

48. Gai X, Tu K, Li C, et al. Histone acetyltransferase PCAF accelerates apoptosis by repressing a GLI1/BCL2/BAX axis in hepatocellular carcinoma. Cell Death Dis 2015;6: e1712.

49. Testa JR, Bellacosa A. AKT plays a central role in tumorigenesis. Proc Natl Acad Sci U S A 2001;98:10983. 